Pacific Journal of Mathematics

ON THE MAXIMUM OF SCALED MULTINOMIAL VARIABLES 


\title{
ON THE MAXIMUM OF SCALED MULTINOMIAL VARIABLES
}

\author{
D. FREEDMAN
}

Suppose $S_{n}$ is a sum of $n$ independent, identically distributed, integer-valued random variables. Let $p_{j}=P\left(S_{n}=j\right)$. Take $k$ independent copies of $S_{n}$, and let $N_{j}$ be the number of these sums which are equal to $j$. In previous papers Persi Diaconis and I studied

$$
\begin{aligned}
& \max _{j} N_{j} \\
& \max _{j}\left(N_{j}-k p_{j}\right) \\
& \max _{j}\left(N_{\jmath}-k \tilde{p}_{j}\right),
\end{aligned}
$$

where $\tilde{p}_{j}$ is the normal approximation to $p_{j}$. Likewise, we have studied the histogram as a density estimator. These problems all have a common structure, namely, determining the asymptotic behavior of the maximum of scaled multinomial variables. The object here is to present a general theorem, flexible enough to cover all the cases mentioned above. The form of this theorem may seem a bit arbitrary at first, but it is suggested by the special cases.

1. Introduction. In this section, the theorem will be stated; the proof is deferred to $\S 3$. Section 2 presents the analogous theorem for normal variables, so as to bring out the main ideas in the proof.

For the main theorem, consider a sequence of multinomial distributions, indexed by $n$. However, this index may be suppressed in later sections, to lighten the notation. At every stage $n$, there are boxes indexed by the integers $j$. Associated with each box is a probability $p_{j}=p_{n j}$; and

$$
\sum_{j=-\infty}^{\infty} p_{n j}=1
$$

The $p_{n j}$ 's will tend to 0 as $n$ grows. At stage $n$, there are $k=k_{n}$ balls; $k_{n} \rightarrow \infty$ as $n \rightarrow \infty$. The balls are dropped independently in turn into the boxes; a ball lands in box $j$ with probability $p_{n j}$. Let $N_{j}=N_{n j}$ be the number of balls which land in box $j$ at stage $n$. Thus, each $N_{n j}$ is binomial with small success probability $p_{n j}$ and large number of trials $k_{n}$. Jointly, the variables $N_{n j}$ for $j=0$, $\pm 1, \cdots$ have a multinomial distribution.

Next, introduce coefficients $\alpha_{j}=\alpha_{n j} \geqq 0$ and $\beta_{j}=\beta_{n j}$. The primary interest is in $\max _{j} V_{n j}$, where 


$$
\begin{aligned}
& V_{n j}=\alpha_{n j} Z_{n j}+\beta_{n j} \sqrt{2 \log 1 / \varepsilon_{n}} . \\
& Z_{n j}=\left(N_{n j}-k_{n} p_{n j}\right) / \sqrt{k_{n} p_{n j}}
\end{aligned}
$$

In (1.1),

$$
\varepsilon=\varepsilon_{n} \longrightarrow 0
$$

is a scale factor for the coefficients, which also have a center $c=c_{n}$. To make this precise, introduce

$$
t_{n j}=\varepsilon_{n}\left(j-c_{n}\right) .
$$

and assume:

the functions $\alpha_{n} \geqq 0$ and $\beta_{n}$ are defined and continuous on a proper compact interval $I$, which does not depend on $n$.

Conditions (1.4) and (1.5) are required to hold uniformly over $j$ with $t_{n j} \in I$. Assume further that

(1.7) $\quad \alpha_{n} \longrightarrow \alpha_{\infty}$ and $\beta_{n} \longrightarrow \beta_{\infty}$ as $n \longrightarrow \infty$, uniformly on $I$.

For $1 \leqq n \leqq \infty$, the function $\alpha_{n}+\beta_{n}$ has a unique global

(1.8) maximum at an interior point $t_{n}$ of $I$, and $\alpha_{n}\left(t_{n}\right)>0$; furthermore, $t_{n} \rightarrow t_{\infty}$.

Conditions (1.7) and (1.8) imply that

$$
\alpha_{n}\left(t_{n}\right) \text { is bounded below by a positive number. }
$$

Assume further that $\alpha_{n}$ and $\beta_{n}$ are locally quadratic at $t_{n}$ : namely, as $t \rightarrow t_{n}$,

$$
\begin{aligned}
& \alpha_{n}(t)=\alpha_{n}\left(t_{n}\right)+\alpha_{n}^{\prime} \cdot\left(t-t_{n}\right)+\frac{1}{2} \alpha_{n}^{\prime \prime} \cdot\left(t-t_{n}\right)^{2}+o\left(t-t_{n}\right)^{2} \\
& \beta_{n}(t)=\beta_{n}\left(t_{n}\right)+\beta_{n}^{\prime} \cdot\left(t-t_{n}\right)+\frac{1}{2} \beta_{n}^{\prime \prime} \cdot\left(t-t_{n}\right)^{2}+o\left(t-t_{n}\right)^{2}
\end{aligned}
$$

where the " 0 " is uniform in $n$. Note that

$$
\begin{aligned}
& \alpha_{n}^{\prime} \longrightarrow \alpha_{\infty}^{\prime}, \quad \alpha_{n}^{\prime \prime} \longrightarrow \alpha_{\infty}^{\prime \prime} \\
& \beta_{n}^{\prime} \longrightarrow \beta_{\infty}^{\prime}, \quad \beta_{n}^{\prime \prime} \longrightarrow \beta_{\infty}^{\prime \prime}
\end{aligned}
$$

In (1.10) and (1.11), it is not necessary to assume differentiability anywhere except at $t_{n}$ : the primes just denote numbers. Necessarily 


$$
\alpha_{n}^{\prime}+\beta_{n}^{\prime}=0 \text {. }
$$

Also, $\alpha_{n}^{\prime \prime}+\beta_{n}^{\prime \prime} \leqq 0$. More is assumed:

$$
\alpha_{n}^{\prime \prime}+\beta_{n}^{\prime \prime}<0 \text { for } 1 \leqq n \leqq \infty
$$

Abbreviate

$$
\rho_{n}^{2}=-\left(\alpha_{n}^{\prime \prime}+\beta_{n}^{\prime \prime}\right) / \alpha_{n}\left(t_{n}\right)>0 ; \text { set } \rho=\rho_{\infty} .
$$

The following growth conditions will be assumed, as $n \rightarrow \infty$ :

$$
\begin{gathered}
\lim \sup _{n \rightarrow \infty} \sum_{j}\left\{p_{n j}: t_{n j} \in I\right\}<1 \\
p_{n j} \cdot\left(\log \frac{1}{\varepsilon_{n}}\right)^{2} \longrightarrow 0 \\
\left(k_{n} p_{n j}\right) /\left(\log \frac{1}{\varepsilon_{n}}\right) \longrightarrow \infty .
\end{gathered}
$$

To avoid "large deviation" terms, a condition stronger than (1.18) is needed:

$$
\left(k_{n} p_{n j}\right) /\left(\log \frac{1}{\varepsilon_{n}}\right)^{3} \longrightarrow \infty \text {. }
$$

Conditions (1.17)-(1.19) are to hold uniformly in $j$ with $t_{n j} \in I$. Note that (1.17)-(1.18) imply

$$
k_{n} /\left(\log \frac{1}{\varepsilon_{n}}\right)^{3} \longrightarrow \infty .
$$

To state the main result, let

$$
w_{n}(x)=\left(2 \log \frac{1}{\varepsilon_{n}}-2 \log \log \frac{1}{\varepsilon_{n}}+x\right)^{1 / 2} .
$$

Let $\Phi$ be the standard normal distribution function, with density $\phi$ :

(1.22) $\Phi(y)=\int_{-\infty}^{y} \phi(u) d u$, where $\phi(u)=\frac{1}{\sqrt{2 \pi}} \exp \left(-\frac{1}{2} u^{2}\right)$.

As usual, $\exp (v)=e^{v}$. Let

$$
M_{n}=\max _{j}\left\{V_{n j}: t_{n j} \in I\right\} \text {, occurring at index } L_{n} \text {. }
$$

Here, $V_{n j}$ is defined by (1.1).

In brief, the main result is that $L_{n}$ and $M_{n}$ are asymptotically independent, $L_{n}$ being asymptotically normal and $M_{n}$ being asymptotically double-exponential.

THEOREM 1.24. Assume (1.1)-(1.23). Let $n \rightarrow \infty$. With proba- 
bility approaching one, $M_{n}=\max _{j}\left\{V_{n j}: t_{n j} \in I\right\}$ is assumed at a unique index $L_{n}$. Furthermore, the chance that

$$
\rho \sqrt{2 \log \frac{1}{\varepsilon_{n}}}\left[\varepsilon_{n}\left(L_{n}-c_{n}\right)-t_{n}\right]<y
$$

and

$$
M_{n}<\alpha_{n}\left(t_{n}\right) w_{n}(x)+\beta_{n}\left(t_{n}\right) \sqrt{2 \log \frac{1}{\varepsilon_{n}}}
$$

converges to

$$
\Phi(y) \cdot \exp \left\{-\frac{1}{2 \rho} e^{-x / 2}\right\}
$$

Now for some heuristic comments about the theorem, especially the assumed error rate $o\left(1 / \log 1 / \varepsilon_{n}\right)$ in $(1.4)-(1.5)$. This error rate is critical. Basically, all the action in $M_{n}$ is over $j$ 's with $t_{n j}$ close to $t_{n}$. So, $M_{n}$ can be crudely approximated as the sum of two terms:

$$
\alpha_{n}\left(t_{n}\right) \max _{j}\left\{Z_{n j}: t_{n j} \text { near } t_{n}\right\}
$$

and

$$
\beta_{n}\left(t_{n}\right) \sqrt{2 \log \frac{1}{\varepsilon_{n}}} .
$$

Both terms are of order $\sqrt{\log 1 / \varepsilon_{n}}$. Changing the coefficients by $o\left(1 / \log 1 / \varepsilon_{n}\right)$ changes $M_{n}$ by $o\left(1 / \sqrt{\log 1 / \varepsilon_{n}}\right)$. Next, consider the asymptotic distribution function for $M_{n}$ in (1.24):

$$
\alpha_{n}\left(t_{n}\right) w_{n}(\cdot)+\beta_{n}\left(t_{n}\right) \sqrt{2 \log \frac{1}{\varepsilon_{n}}} .
$$

This is centered just to the left of

$$
\left[\alpha_{n}\left(t_{n}\right)+\beta_{n}\left(t_{n}\right)\right] \sqrt{2 \log \frac{1}{\varepsilon_{n}}},
$$

which may be large. But the spread is of order

$$
1 / \sqrt{\log \frac{1}{\varepsilon_{n}}}
$$

which is small. So the distribution may move off to infinity, but gets more and more concentrated. And only terms which are $o\left(1 / \sqrt{\log 1 / \varepsilon_{n}}\right)$ can be dropped from $M_{n}$ without affecting its asymptotic behavior.

Now for a comment on $L_{n}$. The action in $M_{n}$ occurs for $j$ 's with $t_{n j}$ near $t_{n}$. At first, it might seem that $O\left(1 / \varepsilon_{n}\right)$ indices $j$ should 
be involved, but this is slightly exaggerated: the right order is

$$
\frac{1}{\varepsilon_{n}} / \sqrt{\log \frac{1}{\varepsilon_{n}}}
$$

Thus, it is necessary to work within shrinking neighborhoods of $t_{\infty}$. Then, it might seem that $\alpha$ and $\beta$ can be treated as constants. Not so, however; the quadratic terms in (1.10)-(1.11) really matter in the asymptotics, as the presence of $\rho$ in the statement of (1.24) should indicate: in effect, however, the linear terms cancel.

Next, a comment on the asymptotic independence. This is a bit surprising. In the vicinity of $t$, the maximum is around

$$
\left[\alpha_{n}(t)+\beta_{n}(t)\right] \sqrt{2 \log \frac{1}{\varepsilon_{n}}}
$$

which diminishes as $t$ moves away from $t_{n}$. Intuition suggests that large values of $L_{n}$ should be accompanied by small values of $M_{n}$. However, this is too hasty. Keeping $t_{n j}$ away from $t_{n}$ makes $V_{n j}$ smaller; but saying that $L_{n}=j$ makes $V_{n j}$ larger. So there is some tension here, and (1.24) shows that the two effects balance.

Finally, a comment on the connection between the multinomial problem and the normal problem discussed in $\S 2$. Formula (1.1) involves the scaled variables

$$
Z_{n j}=\left(N_{n j}-k_{n} p_{n j}\right) / \sqrt{k_{n} p_{n j}}
$$

which are essentially standard normal, and practically independent. So a theorem for normal variables should-and does-go over to the multinomial case. The argument in $\S 2$ is organized so that the estimates can be re-used in $\S 3$. This depends, however, on the growth condition (1.19). If only (1.18) is assumed, the binomials are no longer quite so normal: "large deviations" corrections become relevant. For a discussion of this point, see [2].

Acknowledgment. This paper is an offshoot from an extended collaboration with Persi Diaconis on related problems, and it is a pleasure to acknowledge his help.

2. The normal case. Conditions (1.2)-(1.15) are assumed on the coefficients. Let $U_{j}$ be independent standard normal variables, and

$$
V_{j}=V_{n j}=\alpha_{n j} U_{j}+\beta_{n j} \sqrt{2 \log \frac{1}{\varepsilon_{n}}} .
$$

Define $M_{n}$ and $L_{n}$ as before, by (1.23). Recall (1.8). 
Theorem 2.2. Assume (1.2)-(1.15). Define $V_{n j}$ by (2.1). Assume (1.21)-(1.23). As $n \rightarrow \infty$, the chance that

$$
\rho \sqrt{2 \log \frac{1}{\varepsilon_{n}}}\left[\varepsilon_{n}\left(L_{n}-c_{n}\right)-t_{n}\right]<y
$$

and

$$
M_{n}<\alpha_{n}\left(t_{n}\right) w_{n}(x)+\beta_{n}\left(t_{n}\right) \sqrt{2 \log \frac{1}{\varepsilon_{n}}}
$$

converges to

$$
\Phi(y) \cdot \exp \left[\frac{1}{2 \rho} e^{-1 / 2(x)}\right] .
$$

Without loss of generality, assume

$$
\alpha_{n}\left(t_{n}\right)=1 \text { and } \beta_{n}\left(t_{n}\right)=0 .
$$

Let $\delta$ be a small positive number. It will be shown that j's with $\left|t_{n j}-t_{\infty}\right| \geqq \delta$ make essentially no contribution to the max, because with probability near one, the corresponding $V_{j}$ 's are all less than

$$
(1-\theta) \sqrt{2 \log \frac{1}{\varepsilon_{n}}} .
$$

To make this this precise, only a very weak estimate is needed.

LeMma 2.4. Let $Z_{1}, \cdots, Z_{m}$ be standard normal variables, not necessarily independent. Let $0<a<\infty$. Then

$$
P\left\{\max _{i=1, \cdots, m} Z_{i} \geqq \sqrt{2 \log (a m)\}} \longrightarrow 0\right. \text {. }
$$

Proof. The probability in question is bounded above by

$$
\begin{aligned}
m P\left\{Z_{1}\right. & \geqq \sqrt{2 \log (a m)}\} \approx m \cdot \frac{1}{\sqrt{2 \pi}} \cdot \frac{1}{\sqrt{2 \log a m}} \cdot \exp \left\{-\frac{1}{2} \cdot 2 \log (a m)\right\} \\
& =o(1) .
\end{aligned}
$$

Notation. $y_{n} \approx x_{n}$ means $y_{n} / x_{n} \rightarrow 1$, while $y_{n} \sim x_{n}$ means

$$
0<\liminf _{n \rightarrow \infty} y_{n} / x_{n} \leqq \limsup _{n \rightarrow \infty} y_{n} / x_{n}<\infty \text {. }
$$

Lemma 2.5. Fix any small, positive $\delta$. Let

$$
I_{\delta}=\left\{t: t \in I \text { and }\left|t-t_{\infty}\right| \geqq \delta\right\} .
$$

For some sufficiently small positive $\theta$, the probability that 


$$
\max _{j}\left\{V_{n j}: t_{n j} \in I_{\delta}\right\}<(1-\theta) \sqrt{2 \log \frac{1}{\varepsilon_{n}}}
$$

approaches one as $n \rightarrow \infty$.

Proof. Recall that $\alpha_{n}\left(t_{n}\right)=1$ and $\beta_{n}\left(t_{n}\right)=0$ by the normalization (2.3). From (1.6)-(1.8), there is some $\theta>0$ such that for all large $n$,

$$
(1-5 \theta) \geqq \max _{t}\left\{\alpha_{n}(t)+\beta_{n}(t): t \in I_{\delta}\right\} .
$$

Now use compactness to express $I_{\hat{\delta}}$ as a finite union of intervals $J$ so short that

$$
\alpha_{n}^{J}+\beta_{n}^{J} \leqq 1-4 \theta
$$

where

$$
\alpha_{n}^{I}=\max _{t}\left\{\alpha_{n}(t): t \in J\right\}
$$

and likewise for $\beta_{n}^{J}$.

Fix $J$. By (1.4)-(1.5), for all $n \geqq n_{0}$,

$$
\alpha_{n j} \leqq \alpha_{n}^{J}+\theta \text { and } \beta_{n j} \leqq \beta_{n}^{J}+\theta
$$

for all $j$ with $t_{n j} \in J$. Then

$$
\max _{j}\left\{V_{n j}: t_{n j} \in J\right\}
$$

is bounded above by

$$
\left(\alpha_{n}^{J}+\theta\right) \max _{j}\left\{U_{j} ; t_{n j} \in J\right\}+\left(\beta_{n}^{I}+\theta\right) \sqrt{2 \log \frac{1}{\varepsilon_{n}}} .
$$

By 2.4, with probability near one, the last display is at most

$$
\left(\alpha_{n}^{\prime}+\theta+\beta_{n}^{\prime}+\theta\right) \sqrt{2 \log \frac{1}{\varepsilon_{n}}} \leqq(1-2 \theta) \sqrt{2 \log \frac{1}{\varepsilon_{n}}},
$$

there being only $0\left(1 / \varepsilon_{n}\right)$ indices $j$ with $t_{n j} \in J$. Since there only finitely many $J$ 's, the proof terminates.

Note. In this part of the argument, the error terms in (1.4)(1.5) need only be assumed to be $o(1)$. Also, since $t_{n} \rightarrow t_{\infty}$, for large $n$, if $\left|t_{n j}-t_{n}\right| \geqq \delta$ then $\left|t_{n j}-t_{\infty}\right| \geqq \delta / 2$. Only $j$ 's with $\mid t_{n j}-$ $t_{n} \mid<\delta$ contribute to the max.

Turn now to the $j$ 's with $\left|t_{n j}-t_{n}\right|<\delta$. Here, the argument is more complicated, and a sketch of the idea is given.

For $-\infty \leqq a<b \leqq \infty$, let $I_{a b}$ be the set of $j$ 's with $\left|t_{n j}-t_{n}\right|<$ (2.6) $\delta$ and $a \leqq \rho \sqrt{2 \log \left(1 / \varepsilon_{n}\right)}\left(t_{n j}-t_{n}\right)<b$, and let $M_{a b}=\max _{j}\left\{V_{n j}\right.$ : $\left.j \in I_{a b}\right\}$. 
Despite the notation, $I_{a b}$ and $M_{a b}$ depend on $n$ and $\delta$. It will be proved, among other things, that

$$
P\left\{V_{n j}>w_{n}(x)\right\}=o(1) .
$$

Here and later, " 0 " and " 0 " errors are as $n \rightarrow \infty$, and are uniform over $j$ with $\left|t_{n j}-t_{n}\right|<\delta$.

Clearly, for $-\infty \leqq a<b \leqq c<d \leqq \infty$,

$$
P\left\{M_{a b} \leqq w_{n}(x) \text { and } M_{c d} \leqq w_{n}(y)\right\}=P\left\{M_{a b} \leqq w_{n}(x)\right\} \cdot P\left\{M_{c d} \leqq w_{n}(y)\right\} \text {. }
$$

The factors will be estimated, and appeal made to 2.35 below. For now, only a heuristic argument is given. By (2.7),

$$
\log P\left\{M_{a b} \leqq w_{n}(x)\right\} \doteq-\sum_{j \in I_{a b}} P\left\{V_{n j}>w_{n}(x)\right\} .
$$

The symbol $\doteq$ means approximately equal, and is used only informally. Now

$$
P\left\{V_{n j}>w_{n}(x)\right\}=P\left\{U_{j}>\sqrt{2 \log \frac{1}{\varepsilon_{n}}} \lambda_{n j}(x)\right\},
$$

where

$$
\lambda_{n j}(x)=\frac{w_{n}(x)-\beta_{n j} \sqrt{2 \log \frac{1}{\varepsilon_{n}}}}{\alpha_{n j} \sqrt{2 \log \frac{1}{\varepsilon_{n}}}} .
$$

This $\lambda_{n j}(x)$ is a key technical object in future arguments. To proceed,

$$
\begin{aligned}
& P\left\{U_{j}>\sqrt{2 \log \frac{1}{\varepsilon_{n}}} \lambda_{n j}(x)\right\} \doteq \frac{1}{\sqrt{2 \pi}} \frac{1}{\sqrt{2 \log \frac{1}{\varepsilon_{n}}}} \frac{1}{\lambda_{n j}(x)} \\
& \quad \times \exp \left\{-\left(\log \frac{1}{\varepsilon_{n}}\right) \lambda_{n j}(x)^{2}\right\}
\end{aligned}
$$

It is necessary to estimate $\lambda_{n j}(x)$ : as it turns out,

$$
\begin{aligned}
\lambda_{n j}(x) \doteq 1- & \frac{1}{2}\left(\log \log \frac{1}{\varepsilon_{n}}\right) / \log \frac{1}{\varepsilon_{n}}+\frac{1}{4} x / \log \frac{1}{\varepsilon_{n}} \\
& +\frac{1}{2} \rho^{2}\left(t_{n j}-t_{n}\right)^{2}
\end{aligned}
$$

Now $(1+q)^{2} \doteq 1+2 q$ for small $q$, so

$$
\begin{gathered}
-\left(\log \frac{1}{\varepsilon_{n}}\right) \lambda_{n j}(x)^{2} \doteq-\log \frac{1}{\varepsilon_{n}}+\log \log \frac{1}{\varepsilon_{n}}-\frac{1}{2} x \\
-\frac{1}{2} \rho^{2}\left(2 \log \frac{1}{\varepsilon_{n}}\right)\left(t_{n j}-t_{n}\right)^{2} .
\end{gathered}
$$


Now combine (2.9)-(2.11): the factor $1 / \lambda_{n j}(x)$ on the right in (2.9) is essentially 1 , so

$$
P\left\{U_{j}>\sqrt{2 \log \frac{1}{\varepsilon_{n}}} \lambda_{n j}(x)\right\} \doteq \frac{1}{2 \rho} e^{-x / 2} \cdot \varepsilon^{\prime} \cdot \dot{\phi}\left(u_{j}\right)
$$

where

$$
\begin{aligned}
\varepsilon^{\prime} & =\rho \varepsilon_{n} \sqrt{2 \log \frac{1}{\varepsilon_{n}}} \\
u_{j} & =\rho \sqrt{2 \log \frac{1}{\varepsilon_{n}}}\left(t_{n j}-t_{n}\right)
\end{aligned}
$$

and $\dot{\phi}$ was defined in (1.22) as the standard normal density. Some algebra has been omitted here: the $1 / 2 \rho$ on the right of (2.12) is needed to offset the $\rho \sqrt{2}$ in $\varepsilon^{\prime}$ and get back the $1 / \sqrt{2}$ on the right in (2.9). Continuing from (2.12),

$$
\begin{aligned}
\sum_{j \in I_{a b}} P\left\{U_{j}>\sqrt{2 \log \frac{1}{\varepsilon_{n}}} \lambda_{n j}(x)\right\} & \doteq \frac{1}{2 \rho} e^{-x / 2} \cdot \sum_{j \in I_{a b}} \varepsilon^{\prime} \phi\left(u_{j}\right) \\
& \doteq \frac{1}{2 \rho} e^{-x / 2} \cdot \int_{a}^{b} \phi(u) d u
\end{aligned}
$$

because $u_{j+1}-u_{j}=\varepsilon^{\prime}$.

This argument will now be made rigorous: it is (2.10) and (2.11) which take most of the work. Notice that $\lambda_{n j}(x)$ must be estimated to within $o\left(1 / \log 1 / \varepsilon_{n}\right)$, because its square gets multiplied by $\log 1 / \varepsilon_{n}$. The assumptions are (1.2)-(1.15), (2.1) and (2.3). Two estimates will be needed on $\alpha_{n}$ and $\beta_{n}$; these estimates must be uniform in $n$. The proofs are omitted as routine.

LEMma 2.14. Fix $\eta_{1}>0$. There is a small positive $\delta$ such that for all $n$, and $\left|t-t_{n}\right|<\delta$,

$$
1-\alpha_{n}(t)-\beta_{n}(t)=\frac{1}{2} \rho^{2}\left(1 \pm \eta_{1}\right)\left(t-t_{n}\right)^{2} .
$$

More explicitly, the display means

$$
\frac{1}{2} \rho^{2}\left(1-\eta_{1}\right)\left(t-t_{n}\right)^{2}<1-\alpha_{n}(t)-\beta_{n}(t)<\frac{1}{2} \rho^{2}\left(1+\eta_{1}\right)\left(t-t_{n}\right)^{2} .
$$

LemmA 2.15. Let $(1 / 2) K>1+\sup _{n}\left|\alpha_{n}^{\prime}\right|+1 / 2 \sup _{n}\left|\alpha_{n}^{\prime \prime}\right| . \quad$ There is a small positive $\delta$ such that for all $n$, and $\left|t-t_{n}\right|<\delta$,

$$
\left|\frac{1}{\alpha_{n}(t)}-1\right|<K\left|t-t_{n}\right| \text {. }
$$


It is now time to estimate the $\lambda_{n j}(x)$ defined in (2.8), making (2.10)-(2.11) precise.

LEMMA 2.16. Fix $\eta>0$. For small positive $\delta$, the following estimates will apply as $n \rightarrow \infty$, uniformly over $j$ with $\left|t_{n j}-t_{n}\right|<\delta$, and uniformly over $x$ in compact intervals.

(a) $\left|\lambda_{n j}(x)-1\right|<\eta+o(1)$

(b) $\left(\log 1 / \varepsilon_{n}\right) \lambda_{n j}(x)^{2} \leqq \log 1 / \varepsilon_{n}-\log \log 1 / \varepsilon_{n}+(x+\eta) / 2+\left(\rho^{2} / 2\right)\left(1^{\prime}+\right.$ $\eta)^{2}\left(2 \log 1 / \varepsilon_{n}\right)\left(t_{n j}-t_{n}\right)^{2}+o(1)$

(c) $\left(\log 1 / \varepsilon_{n}\right) \lambda_{n j}(x)^{2} \geqq \log 1 / \varepsilon_{n}-\log \log 1 / \varepsilon_{n}+(x-\eta) / 2+\left(\rho^{2} / 2\right)(1-$ $\eta)^{2}\left(2 \log 1 / \varepsilon_{n}\right)\left(t_{n j}-t_{n}\right)^{2}+o(1)$

Proof. Choose $\delta$ so small that the estimates in (2.14)-(2.15) apply, with $\eta_{1}$ to be chosen later. Also choose $\delta$ so small that

$$
\frac{3}{4}<\alpha_{n}(t)<\frac{5}{4} \text { and }-\frac{1}{4}<\beta_{n}(t)<\frac{1}{4} \text { for }\left|t-t_{n}\right|<\delta .
$$

And so, for $n \geqq n_{0}$,

8) $\frac{1}{2}<\alpha_{n j}<\frac{3}{2}$ and $-\frac{1}{2}<\beta_{n j}<\frac{1}{2}$ for $\left|t_{n j}-t_{n}\right|<\delta$.

In (2.8), replace $\beta_{n j}$ by $\beta_{n}\left(t_{n j}\right)$ and $\alpha_{n j}$ by $\alpha_{n}\left(t_{n j}\right)$. This gives a new quantity, to be denoted by $\lambda_{n j}^{*}(x)$. The first job is to show that

$$
\lambda_{n j}(x)=\lambda_{n j}^{*}(x)+o\left(1 / \log \frac{1}{\varepsilon_{n}}\right) .
$$

However, the first move only caused an error of

$$
\left[\beta_{n j}-\beta_{n}\left(t_{n j}\right)\right] / \alpha_{n j}=o\left(1 / \log \frac{1}{\varepsilon_{n}}\right)
$$

by (2.18) and assumption (1.5). Likewise, the second move only caused an error of

$$
\left[\frac{w_{n}(x)}{\sqrt{2 \log \frac{1}{\varepsilon_{n}}}}-\beta_{n}\left(t_{n j}\right)\right]\left[\frac{1}{\alpha_{n j}}-\frac{1}{\alpha_{n}\left(t_{n j}\right)}\right]=o\left(1 / \log \frac{1}{\varepsilon_{n}}\right) .
$$

This completes the proof of (2.19).

To proceed, let

$$
\begin{gathered}
r_{n}=\left(\log \log \frac{1}{\varepsilon_{n}}\right) /\left(\log \frac{1}{\varepsilon_{n}}\right) \text { and } s_{n}(x)=x /\left(2 \log \frac{1}{\varepsilon_{n}}\right) \\
q_{n j}(x)=\lambda_{n j}^{*}(x)-1
\end{gathered}
$$




$$
\begin{aligned}
& =\frac{\left[1-r_{n}+s_{n}(x)\right]^{1 / 2}-\beta_{n}\left(t_{n j}\right)}{\alpha_{n}\left(t_{n j}\right)}-1 \\
& =\frac{\left[1-r_{n}+s_{n}(x)\right]^{1 / 2}-\alpha_{n}\left(t_{n j}\right)-\beta_{n}\left(t_{n j}\right)}{\alpha_{n}\left(t_{n j}\right)} .
\end{aligned}
$$

This trivial bit of algebra is the key to the proof. Expand the square root and use (2.14):

$$
\begin{aligned}
q_{n j}(x) & =\frac{1-\alpha_{n}\left(t_{n j}\right)-\beta_{n}\left(t_{n j}\right)-\frac{1}{2} r_{n}+\frac{1}{2} s_{n}(x)}{\alpha_{n}\left(t_{n j}\right)}+o\left(1 / \log \frac{1}{\varepsilon_{n}}\right) \\
& =\frac{\frac{1}{2} \rho^{2}\left(1 \pm \eta_{1}\right)\left(t_{n j}-t_{n}\right)^{2}-\frac{1}{2} r_{n}+\frac{1}{2} s_{n}(x)}{\alpha_{n}\left(t_{n j}\right)}+o\left(1 / \log \frac{1}{\varepsilon_{n}}\right) .
\end{aligned}
$$

Now use (2.15) to estimate $\alpha_{n}\left(t_{n j}\right)^{-1}-1$ :

$$
\begin{aligned}
q_{n j}(x)= & \frac{1}{2} \rho^{2}\left(1 \pm \eta_{1}\right)\left(t_{n j}-t_{n}\right)^{2}-\frac{1}{2} r_{n}+\frac{1}{2} s_{n}(x)+\tau_{1}+\tau_{2} \\
& +\tau_{3}+o\left(1 / \log \frac{1}{\varepsilon_{n}}\right)
\end{aligned}
$$

where

$$
\begin{aligned}
& \left|\tau_{1}\right|<\frac{1}{2} \rho^{2}\left(1+\eta_{1}\right)\left(t_{n j}-t_{n}\right)^{2} \cdot K\left|t_{n j}-t_{n}\right| \\
& \left|\tau_{2}\right|<\frac{1}{2} r_{n} \cdot K\left|t_{n j}-t_{n}\right| \\
& \left|\tau_{3}\right|<\frac{1}{2} s_{n}(|x|) \cdot K\left|t_{n j}-t_{n}\right| .
\end{aligned}
$$

Now $\left|t_{n j}-t_{n}\right|<\delta$ by assumption, and $K \delta$ gets small with $\delta$, so $\tau_{1}$ merges into the first term: for small enough $\delta$,

$$
\frac{1}{2} \rho^{2}\left(1 \pm \eta_{1}\right)\left(t_{n j}-t_{n}\right)^{2}+\tau_{1}=\frac{1}{2} \rho^{2}\left(1 \pm 2 \eta_{1}\right)\left(t_{n j}-t_{n}\right)^{2} .
$$

This uses (1.14)-(1.15) to force $\rho>0$. Likewise, $\tau_{3}$ merges into $s_{n}(x)$ :

$$
\frac{1}{2} s_{n}(x)+\tau_{3}=\frac{1}{2} s_{n}(x \pm \eta) .
$$

This leaves $\tau_{2}$. With respect to this error, the claim is

$$
\tau_{2}=o\left(t_{n j}-t_{n}\right)^{2}+o\left(1 / \log \frac{1}{\varepsilon_{n}}\right) .
$$


Indeed, if $\left|t_{n j}-t_{n}\right| \leqq 1 /\left(\log 1 / \varepsilon_{n}\right)^{1 / 2}$ then

$$
\left|\tau_{2}\right| \leqq \frac{1}{2} K\left(\log \log \frac{1}{\varepsilon_{n}}\right) /\left(\log \frac{1}{\varepsilon_{n}}\right)^{3 / 2}=o\left(1 / \log \frac{1}{\varepsilon_{n}}\right) .
$$

On the other hand, if $\left|t_{n j}-t_{n}\right|>1 /\left(\log 1 / \varepsilon_{n}\right)^{1 / 2}$ then

$$
\left|\tau_{2}\right| /\left(t_{n j}-t_{n}\right)^{2} \leqq \frac{1}{2} K\left(\log \log \frac{1}{\varepsilon_{n}}\right) /\left(\log \frac{1}{\varepsilon_{n}}\right)^{1 / 2}=o(1) .
$$

This completes the proof of (2.20). But (2.20) shows that $\tau_{2}$ merges into the lead term as well:

$$
\frac{1}{2} \rho^{2}\left(1 \pm 2 \eta_{1}\right)\left(t_{n j}-t_{n}\right)^{2}+\tau_{2}=q_{n j}^{*}(x)+o\left(1 / \log \frac{1}{\varepsilon_{n}}\right)
$$

where

$$
q_{n j}^{*}(x)=\frac{1}{2} \rho^{2}\left(1 \pm 3 \eta_{1}\right)\left(t_{n j}-t_{n}\right)^{2}
$$

Combining this with (2.19): for small $\delta$,

$$
\lambda_{n j}(x)=1+q_{n j}^{*}(x)+o\left(1 / \log \frac{1}{\varepsilon_{n}}\right) .
$$

Now (2.22) proves claim (a) of the lemma, because $\left(t_{n j}-t_{n}\right)^{2} \leqq$ $\delta^{2}$, so $q_{n j}^{*}(x)$ as defined in $(2.21)$ is small with $\delta$. Turning to claims (b) $-(\mathrm{c})$,

$$
\lambda_{n j}(x)^{2}=1+2 q_{n_{j}}^{*}(x)+q_{n j}^{*}(x)^{2}+o\left(1 / \log \frac{1}{\varepsilon_{n}}\right) .
$$

But $q_{n j}^{*}(x)^{2}$ merges into $q_{n j}^{*}(x)$, because the latter is small: referring to $(2.21)$,

$$
2 q_{n j}^{*}(x)+q_{n j}^{*}(x)^{2}=\rho^{2}\left(1 \pm 4 \eta_{1}\right)\left(t_{n j}-t_{n}\right)^{2} .
$$

To complete the proof, choose $\eta_{1}$ so that

$$
(1-\eta)^{2}<1-4 \eta_{1}<1+4 \eta_{1}<(1+\eta)^{2} .
$$

This made (2.10)-(2.11) rigorous. Next, take up (2.13). Introduce

$$
\psi_{n j}(x)=\left(4 \pi \log \frac{1}{\varepsilon_{n}}\right)^{-1 / 2} \lambda_{n j}(x)^{-1} \cdot \exp \left\{-\left(\log \frac{1}{\varepsilon_{n}}\right) \lambda_{n j}(x)^{2}\right\} .
$$

LEMMA 2.24. If $\delta$ is small, $\psi_{n j}(x)=o(1)$ as $n \rightarrow \infty$, uniformly over $j$ with $\left|t_{n j}-t_{n}\right|<\delta$, and uniformly over $x$ in compact intervals.

Proof. This is immediate from (2.16a), because $\lambda_{n j}(x)$ is essen- 
tially one, and $\log 1 / \varepsilon_{n}$ goes to infinity.

LEMMA 2.25. Fix $\eta>0$. If $\delta$ is small, the following estimates apply as $n \rightarrow \infty$, uniformly over extended real $a$ and $b$, and $x$ in compact intervals.

(a) $\sum_{j \in I_{a b}} \psi_{n j}(x)$ is bounded below by

$$
\frac{1}{(1+\eta)^{2}}\{\Phi[b(1+\eta)]-\Phi[a(1+\eta)]\} \cdot \frac{1}{2 \rho} \cdot \exp \left\{-\frac{1}{2}(x+\eta)\right\}+o(1) .
$$

(b) $\sum_{j \in I_{a b}} \psi_{n j}(x)$ is bounded above by

$$
\frac{1}{(1-\eta)^{2}}\{\Phi[b(1-\eta)]-\Phi[a(1-\eta)]\} \cdot \frac{1}{2 \rho} \cdot \exp \left\{-\frac{1}{2}(x-\eta)\right\}+o(1) .
$$

Proof. This follows from (2.16). Claim (a) will be argued in some detail, and (b) is similar. Define

$$
\begin{aligned}
& \varepsilon^{\prime}=\rho(1+\eta) \varepsilon_{n}\left(2 \log \frac{1}{\varepsilon_{n}}\right)^{1 / 2} \\
& u_{j}=\rho(1+\eta)\left(2 \log \frac{1}{\varepsilon_{n}}\right)\left(t_{n j}-t_{n}\right) .
\end{aligned}
$$

The dependence of $\varepsilon^{\prime}$ and $u_{j}$ on $n$ is suppressed. Recall that $\dot{\phi}$ is the standard normal density. As (2.16a) implies,

$$
\lambda_{n j}(x)^{-1} \geqq[1+o(1)] /(1+\eta) .
$$

As (2.16b) now implies, $\psi_{n j}(x)$ is bounded below by

$$
[1+o(1)] \frac{1}{(1+\eta)^{2}} \cdot \frac{1}{2 \rho} \cdot \exp \left\{-\frac{1}{2}(x+\eta)\right\} \cdot \varepsilon^{\prime} \phi\left(u_{j}\right) .
$$

The " $o(1)$ " is as $n \rightarrow \infty$, and is uniform over $j$ with $\left|t_{n j}-t_{n}\right|<\delta$, for small enough $\delta$.

Consider

$$
\sum_{j \in I_{a b}} \varepsilon^{\prime} \dot{\phi}\left(u_{j}\right) .
$$

Suppose $0 \leqq a<b<\infty$. Clearly, $u_{j+1}-u_{j}=\varepsilon^{\prime}$; and $\phi$ is monotone decreasing on $[0, \infty)$. So

$$
\varepsilon^{\prime} \phi\left(u_{j}\right)>\int_{u_{j}}^{u_{j+1}} \phi(u) d u .
$$

As definition (2.6) shows, provided $n$ is sufficiently large, $j \in I_{a b}$ iff $a(1+\eta) \leqq u_{j}<b(1+\eta)$. Then the sum in (2.26) is bounded below by

$$
\int_{a(1+\eta)+\varepsilon^{\prime}}^{b(1+\eta)} \dot{\phi}(u) d u \text {. }
$$


The $\varepsilon^{\prime}$ in the lower limit of the integral takes care of an edge effect: $j$ is discrete, $u$ is continuous. Clearly, the displayed integral exceeds

$$
\int_{a(1+\eta)}^{b(1+\eta)} \phi(u) d u-\int_{0}^{\varepsilon^{\prime}} \phi(u) d u
$$

This completes the argument for (a) in case $0 \leqq a<b<\infty$; the case $b=\infty$ then follows; the case $-\infty \leqq a<b \leqq 0$ is symmetric; and the general case follows by addition. This disposes of claim (a), and $(b)$ is similar.

The $\eta$ in (2.16) and (2.25) is a nuisance. Over the interesting $j$, with

$$
\rho \sqrt{2 \log \frac{1}{\varepsilon_{n}}}\left|t_{n j}-t_{n}\right|<B<\infty,
$$

the argument in (2.16) is sharp enough to establish the results with $\eta=0$. However, something needs to be done to cover the $j$ 's with, for instance,

$$
\begin{aligned}
B<\rho \sqrt{2 \log \frac{1}{\varepsilon_{n}}}\left(t_{n j}-t_{n}\right) & <\infty \\
\left(t_{n j}-t_{n}\right) & <\delta .
\end{aligned}
$$

To do that, $\eta$ was needed. Now, however, this technical nuisance can be eliminated. The interval $I_{a b}$ was defined in (2.6), and depends on $\delta$. This dependence matters in the next result, so the interval will be denoted $I_{a b}(\delta)$.

Proposition 2.27. If $\delta$ is small, uniformly over extended real $a$ and $b$, and uniformly over $x$ in compact intervals, as $n \rightarrow \infty$,

$$
\sum_{j \in I_{a b}(\hat{\partial})} \psi_{n j}(x) \longrightarrow[\Phi(b)-\Phi(a)] \frac{1}{2 \rho} \exp \left\{-\frac{1}{2} x\right\} .
$$

Proof. Denote the left side of (2.28) by $S_{n}(a, b, \delta, x)$; and the right side by $T(a, b, x)$. The first thing to show is that the tails don't matter. Fix $\eta$ in (2.25) at any convenient value, say, $\eta=1 / 2$. This generates a corresponding $\delta$, for which the estimate in (2.25b) is valid. This is the $\delta$ to use. Let $0<B<\infty$, but large. Then

$$
\limsup _{n \rightarrow \infty} S_{n}(B, \infty, \delta, x)
$$

is bounded above according to (2.25b), and this bound is small for large $B$. Likewise for 


$$
\limsup _{n \rightarrow \infty} S_{n}(-\infty,-B, \delta, x)
$$

Let

$$
\hat{a}=(-B) \wedge a \text { and } \hat{b}=b \wedge B .
$$

Then $S_{n}(a, b, \delta, x)$ is bounded below by $S_{n}(\hat{a}, \hat{b}, \delta, x)$, and above by

$$
S_{n}(\hat{a}, \hat{b}, \delta, x)+S_{n}(-\infty,-B, \delta, x)+S_{n}(B, \infty, \delta, x) .
$$

As a result, it is only necessary to prove the lemma for $a$ and $b$ with

$$
-B \leqq a<b \leqq B
$$

Now, if $j \in I_{a b}(\delta)$, then $j \in I_{a b}\left(\delta^{\prime}\right)$ for $n \geqq n_{0}$, where $n_{0}$ depends on $\delta^{\prime}$ but on $j$. Here, $\delta^{\prime}$ is positive but much smaller than $\delta$. As a result, (2.25) applies with $\eta$ arbitrarily small.

Step (2.9) in the heuristic argument is easy to rigorize, in view of (2.16).

For small $\delta$ : uniformly in $j$ with $\left|t_{n j}-t_{n}\right|<\delta$, as $n \rightarrow \infty$,

$$
P\left\{U_{j}>\sqrt{2 \log \frac{1}{\varepsilon_{n}}} \lambda_{n j}(x)\right\}=[1+o(1)] \psi_{n j}(x) .
$$

Now (2.13) can be finished.

Proposition 2.30. If $\delta$ is small:

(a) $P\left\{U_{j}>\sqrt{2 \log \left(1 / \varepsilon_{n}\right)} \lambda_{n j}(x)\right\}=o(1)$ as $n \rightarrow \infty$, uniformly over $j$ with $\left|t_{n j}-t_{n}\right|<\delta$, and uniformly over $x$ in compact intervals;

(b) $\sum_{j \in I_{a b}} P\left\{U_{j}>\sqrt{2 \log \left(1 / \varepsilon_{n}\right)} \lambda_{n j}(x)\right\}$ converges to

$$
[\Phi(b)-\Phi(a)] \cdot \frac{1}{2 \rho} \cdot e^{-x / 2}
$$

as $n \rightarrow \infty$, uniformly over extended real numbers $a$ and $b$, and $x$ in compact intervals.

Proof. Claim (a) is immediate from (2.29) and (2.24). Likewise, claim (b) is immediate from (2.29) and (2.27).

This completes the rigorous discussion of (2.13). Recall $M_{a b}$ from (2.6). The next step is to determine the joint distribution of $M_{a b}$ and $M_{c d}$.

Proposition 2.31. If $\delta$ is small: uniformly over $a, b, c, d$ with 
$-\infty \leqq a<b \leqq c<d \leqq \infty$, and uniformly over $x, y$ in compact intervals,

$$
P\left\{M_{a b} \leqq w_{n}(x) \quad \text { and } \quad M_{c d} \leqq w_{n}(y)\right\}
$$

converges to $\exp \{Q(x, y)\}$ as $n \rightarrow \infty$, where

$$
-2 \rho Q(x, y)=[\Phi(b)-\Phi(a)] e^{-(1 / 2) x}+[\Phi(d)-\Phi(c)] e^{-(1 / 2) y} .
$$

Proof. Clearly, the logarithm of the probability is

$$
\sum_{j \in \perp_{a b}} \log P\left\{V_{n j} \leqq w_{n}(x)\right\}+\sum_{J \in I_{c d}} \log P\left\{V_{n j} \leqq w_{n}(y)\right\} .
$$

Take the first sum, for instance. Definition (2.8) of $\lambda_{n j}(x)$ was set up so that

$$
V_{n j} \leqq w_{n}(x) \text { iff } \quad U_{j} \leqq \sqrt{2 \log \frac{1}{\varepsilon_{n}}} \lambda_{n j}(x) .
$$

Expanding $\log p=\log [1-(1-p)] \doteq-(1-p)$, the first sum in (2.32) can be estimated as

$$
-[1+o(1)] \sum_{j \in \perp_{a b}} P\left\{U_{j}>\sqrt{2 \log \frac{1}{\varepsilon_{n}}} \lambda_{n j}(x)\right\} \longrightarrow-[\Phi(b)-\Phi(a)] \frac{1}{2 \rho} e^{-(1 / 2) x},
$$

using (2.30). The other sum is similar.

In (2.31), the index $j$ was restricted so that $\left|t_{n j}-t_{n}\right|<\delta$. This was part of the definition of $I_{a b}=I_{a b}(\delta)$, even for infinite $a$ and $b$, in (2.6). As a result, $M_{a b}$ depends on $\delta$ too; write $M_{a b}(\delta)$ to indicate this dependence. The restriction on $j$ was necessary, to make the estimates in (2.16). However, it can now be eliminated.

COROLlARY 2.33. The conclusions of (2.31) apply, whatever $\delta$ may be.

Proof. Let $\delta$ be small, so that (2.31) applies, and let $\delta^{\prime}$ be large. Let

$$
M^{*}=\max \left\{V_{n j}:\left|t_{n j}-t_{n}\right| \geqq \delta\right\}
$$

Clearly,

$$
M_{a b}(\delta) \leqq M_{a b}\left(\delta^{\prime}\right) \leqq M_{a b}(\delta) \vee M^{*}
$$

But (2.5) and (2.31) show

$$
P\left\{M^{*} \leqq(1-\theta) \sqrt{2 \log \frac{1}{\varepsilon_{n}}} \leqq M_{a b}(\delta)\right\} \longrightarrow 1,
$$


so

$$
P\left\{M_{a b}\left(\delta^{\prime}\right)=M_{a b}(\delta)\right\} \longrightarrow 1 .
$$

In particular, $\delta$ can be chosen so large that $\left\{t:\left|t-t_{n}\right|<\delta\right\}$ includes all of $I$. The proof of Theorem 2.2 is then accomplished by appeal to (2.35) below.

It is helpful, at times, to take $\max _{j} V_{n j}$ not only over the $j$ with $t_{n j} \in I$, but over all integers $j$. This can be done if $\alpha_{n j}$ and $\beta_{n j}$ are defined for all $j$, and $j$ 's with $t_{n j} \notin I$ do not count. This can be made precise, as follows.

Corollary 2.34. Assume (1.2)-(1.15). Define $V_{n j}$ by (2.1), for all integers $j$. Assume (1.21)-(1.23). Assume further that for some $\theta>0$,

$$
\max _{j}\left\{V_{n j}: t_{n j} \notin I\right\}<(1-\theta) \sqrt{2 \log \frac{1}{\varepsilon_{n}}}
$$

with probability approaching one as $n \rightarrow \infty$. Then the conclusions of (2.2) apply to

$$
M_{n}=\max _{j}\left\{V_{n j}: \text { all integer } j\right\} .
$$

Proof. Use the argument of (2.33).

Note. The condition here is that $\alpha_{n j}$ should get small, or $\beta_{n j}$ should become large and negative, or both, as $j \rightarrow \pm \infty$.

Theorem 2.35 below will be used repeatedly, and so it is given here in some generality.

Framework for 2.35. Let $V_{n j}$ be a random variable, defined for integers $j$ in a finite, non-empty (non-random) interval $J_{n}$. For (2.2), take $J_{n}$ to be the set of $j$ 's with $t_{n j} \in I$, and $V_{n j}$ is defined by (2.1). Let $v_{n}(y)$ be a strictly increasing function of $y$, with $v_{n}(-\infty)=-\infty$ and $v_{n}(\infty)=\infty$. For (2.2),

$$
v_{n}(y)=\rho \sqrt{2 \log \frac{1}{\varepsilon_{n}}}\left[\varepsilon_{n}\left(y-c_{n}\right)-t_{n}\right] .
$$

Likewise, let $w_{n}(x)$ be a continuous and strictly increasing function of $x$, with $w_{n}(-\infty)=-\infty$ and $w_{n}(\infty)=\infty$. For (2.2), this function is defined by (1.21) down to

$$
x=-2 \log \frac{1}{\varepsilon_{n}}+2 \log \log \frac{1}{\varepsilon_{n}} ;
$$

it may be extended back to $-\infty$ in any convenient way, subject to the conditions given above. Let 


$$
\begin{aligned}
& I_{a b}^{n}=\left\{j: j \in J_{n} \text { and } v_{n}(a) \leqq j<v_{n}(b)\right\} \\
& M_{a b}^{n}=\max _{j}\left\{V_{n j}: j \in I_{a b}^{n}\right\}
\end{aligned}
$$

with $M_{a b}^{n}=-\infty$ if $I_{a b}^{n}$ is empty. Let

$$
M_{n}=\max _{j}\left\{V_{n j}: j \in J_{n}\right\}
$$

with $L_{n}$ being the leftmost $j$ at which the $\max$ occurs, and $\hat{L}_{n}$ the rightmost. Let $\Phi$ be a distribution function: for (2.2), the standard normal. Let $\psi$ be a monotone increasing function on $(-\infty, \infty)$, with $\psi(-\infty)=-\infty$, and $\psi(\infty)=0$; suppose too that $\psi$ has a continuous derivative $\psi^{\prime}$. For (2.2), take $\psi(x)=-(1 / 2 \rho) e^{-x / 2}$.

THEOREM 2.35. Under the conditions given above, suppose that for $-\infty \leqq a<b \leqq c<d \leqq \infty$

$$
P\left\{M_{a b}^{n} \leqq w_{n}(x) \quad \text { and } \quad M_{c d}^{n} \leqq w_{n}(y)\right\}
$$

converges to $\exp \{Q(x, y)\}$ as $n \rightarrow \infty$, where

$$
Q(x, y)=[\Phi(b)-\Phi(a)] \psi(x)+[\Phi(d)-\Phi(c)] \psi(y)
$$

Then

$$
P\left\{v_{n}(a) \leqq L_{n} \leqq \hat{L}_{n} \leqq v_{n}(b) \text { and } M_{n} \leqq w_{n}(x)\right\}
$$

converges to

$$
[\Phi(b)-\Phi(a)] \cdot \exp \{\psi(x)\}
$$

Proof. Begin with the case $a=-\infty$. Since $v_{n}(-\infty)=-\infty$, there is no condition on $L_{n}$. Now

$$
\hat{L}_{n} \leqq v_{n}(b) \text { and } M_{n}>w_{n}(x)
$$

iff

$$
X_{n}>x \text { and } X_{n}>Y_{n}
$$

where

$$
X_{n}=w_{n}^{-1}\left(M_{-\infty b}^{n}\right) \quad \text { and } \quad Y_{n}=w_{n}^{-1}\left(M_{b \infty}^{n}\right) \text {. }
$$

By assumption, $w_{n}$ is strictly increasing, and its range is the whole line, so $w_{n}^{-1}$ is well defined.

As is given in the statement of the proposition, $\left(X_{n}, Y_{n}\right)$ converges in law to $(X, Y)$, where

$$
P\{X \leqq x \text { and } Y \leqq y\}=\exp \{Q(x, y)\} .
$$

In particular, $X$ has the probability density

$$
\Phi(b) \psi^{\prime}(u) \exp \{\Phi(b) \psi(u)\}
$$


while

$$
P\{Y<u\}=\exp \{[1-\Phi(b)] \psi(u)\} ;
$$

and $X, Y$ are idependent. Note that $\psi$ is negative, but $\psi^{\prime}$ is positive. Now

$$
P\left\{X_{n}>x \text { and } X_{n}>Y_{n}\right\} \longrightarrow P\{X>x \text { and } X>Y\} .
$$

The limiting probability is

$$
\begin{aligned}
\left.\int_{x}^{\infty} \Phi(b) \psi^{\prime}(u) \exp \{\Phi(b) \psi(u)]\right\} \cdot \exp \{[1-\Phi(b)] \psi(u)\} d u \\
\quad=\Phi(b) \int_{x}^{\infty} \psi^{\prime}(u) \exp \{\psi(u)\} d u \\
\quad=\Phi(b)[\exp \{\psi(\infty)\}-\exp \{\psi(x)\}] \\
\quad=\Phi(b)[1-\exp \{\psi(x)\}]
\end{aligned}
$$

This proves the theorem when $a=-\infty$ and $-\infty<b<\infty$. A similar argument goes through when $-\infty<a<\infty$ and $b=\infty$. In particular, $L_{n}$ and $\hat{L}_{n}$ have the same asymptotic distribution, namely, the law of $v_{n}^{-1}\left(L_{n}\right)$ converges weak-star to $\Phi$, and likewise for $v_{n}^{-1}\left(\hat{L}_{n}\right)$. Since $v_{n}^{-1}\left(L_{n}\right) \leqq v_{n}^{-1}\left(\hat{L}_{n}\right)$, it follows that

$$
v_{n}^{-1}\left(\hat{L}_{n}\right)-v_{n}^{-1}\left(L_{n}\right) \longrightarrow 0 \text { in probability. }
$$

The balance of the argument is omitted as routine.

3. The multinomial case. In this section, Theorem 1.24 will be proved. We are back in the multinomial situation: (1.1)-(1.23) are in force. Without further loss of generality, assume the normalization (2.3). Again, let $\delta$ be a small positive number. The $j$ 's with $\left|t_{n j}-t_{\infty}\right| \geqq \delta$ make essentially no contribution to the $\max$, because with probability near one, the corresponding $V_{n j}$ 's are all less than

$$
(1-\theta) \sqrt{2 \log \frac{1}{\varepsilon}} .
$$

This will be seen in (3.3). Here $\varepsilon=\varepsilon_{n}$; the subscript $n$ was dropped to lighten the notation.

Lemma 3.1. Let $\zeta>0$. Then

$$
P\left\{\max _{j} Z_{n j}>(1+\zeta) \sqrt{\log \frac{1}{\varepsilon}}\right\} \longrightarrow 0
$$

as $n \rightarrow \infty$, the max being taken over all $j$ with $t_{n j} \in I$.

Proof. The chance that 


$$
N_{j}-k p_{j}>(1+\zeta) \sqrt{k p_{j}} \sqrt{2 \log \frac{1}{\varepsilon}}
$$

is at most

$$
\exp \left\{-\left(\log \frac{1}{\varepsilon}\right)(1+\zeta)^{2} \gamma\right\}
$$

where

$$
\gamma=1 /\left[1+(1+\zeta) \sqrt{\left(2 \log \frac{1}{\varepsilon}\right) / k p_{j}}\right]
$$

is uniformly close to 1 by condition (1.18). Eventually, $\gamma>1 /(1+\zeta)$, and then the probability of the event (3.2) will be bounded above by

$$
\exp \left\{-\left(\log \frac{1}{\varepsilon}\right)(1+\zeta)\right\}=\varepsilon^{1+\zeta}
$$

However, there are only $0(1 / \varepsilon)$ indices $j$ with $t_{n j} \in I$. The version of Bernstein's inequality used above appears as theorem (4) in [5].

LEMMA 3.3. Fix any small positive $\delta$. Let

$$
I_{\delta}=\left\{t: t \in I \text { and }\left|t-t_{\infty}\right| \geqq \delta\right\} .
$$

For sufficiently small positive $\theta$, the probability that

$$
\max _{j}\left\{V_{n j}: t_{n j} \in I_{j}\right\}<(1-\theta) \sqrt{2 \log \frac{1}{\varepsilon}}
$$

approaches one as $n$ tends to infinity.

Proof. Argue exactly as in (2.5), but use (3.1) instead of (2.4). For $n \geqq n_{0}$,

$$
\max _{j}\left\{V_{n j}: t_{n j} \in J\right\}
$$

is bounded above by

$$
\left(\alpha_{n}^{J}+\theta\right) \max _{j}\left\{Z_{n j}: t_{n j} \in J\right\}+\left(\beta_{n}^{J}+\theta\right) \sqrt{2 \log \frac{1}{\varepsilon}} .
$$

By (3.1), with probability approaching one, the last display is at most

$$
\begin{aligned}
& {\left[\left(\alpha_{n}^{I}+\theta\right)(1+\zeta)+\left(\beta_{n}^{J}+\theta\right)\right] \sqrt{2 \log \frac{1}{\varepsilon}}} \\
& \quad<(1+\zeta)\left(\alpha_{n}^{I}+\beta_{n}^{I}+2 \theta\right) \sqrt{2 \log \frac{1}{\varepsilon}}
\end{aligned}
$$




$$
\begin{aligned}
& <(1+\zeta)(1-2 \theta) \sqrt{2 \log \frac{1}{\varepsilon}} \\
& <(1-\theta) \sqrt{2 \log \frac{1}{\varepsilon}}
\end{aligned}
$$

for small $\zeta$.

Note. Again, in this part of the argument, the error terms in (1.4)-(1.5) need only be assumed to be $o(1)$. The result disposes of the $j$ with $\left|t_{n j}-t_{n}\right| \geqq \delta$.

It is now time to deal with the $j$ 's for which $\left|t_{n j}-t_{n}\right|<\delta$. Define $I_{a b}$ and $M_{a b}$ by (2.6), with $V_{n j}$ from (1.1). Despite the notation, $I_{a b}$ and $M_{a b}$ depend on $n$ and on $\delta$. This will be made explicit only when it matters.

Proposition 3.4. Assume (1.1)-(1.23). If $\delta$ is small, and $-\infty \leqq a<b \leqq c<d \leqq \infty$, then

$$
P\left\{M_{a b} \leqq w_{n}(x) \quad \text { and } \quad M_{c d} \leqq w_{n}(y)\right\}
$$

converges to $\exp \{Q(x, y)\}$ as $n \rightarrow \infty$, where

$$
-2 \rho Q(x, y)=[\Phi(b)-\Phi(a)] e^{-(1 / 2) x}+[\Phi(d)-\Phi(c)] e^{-(1 / 2) y} .
$$

Granting (3.4), the condition on $\delta$ can be eliminated by (3.3), just as in (2.33). Then the proof of (1.24) can be completed by appealing to (2.35). Thus, (1.24) reduces to (3.4). Before going on to the proof of (3.4), note that it may sometimes be helpful to take $\max _{j} V_{n j}$ not only over the $j$ with $t_{n j} \in I$, but over all integer $j$. This can be done, for $\alpha_{n j}, \beta_{n j}, p_{n j}$ and $N_{n j}$ are defined for all $j$, as is $V_{n j}$ by (1.1).

Corollary 3.5. Assume (1.1)-(1.23). Suppose further that for some $\theta>0$,

$$
\max _{j}\left\{V_{n j}: t_{n j} \notin I\right\}<(1-\theta) \sqrt{2 \log \frac{1}{\varepsilon_{n}}}
$$

with probability approaching one as $n$ tends to infinity. Then the conclusions of (1.24) apply as well to

$$
M_{n}=\max _{j}\left\{V_{n j}: \text { all integer } j\right\} \text {. }
$$

Proof. Use the argument of (2.33).

Turn now to the proof of (3.4). It will be necessary to estimate the probability above and below. The upper bound is easier. In 
essence, an inequality of Mallows (1968) shows that the probability is at most

$$
\prod_{j \in I_{a b}} P\left\{V_{n j} \leqq w_{n}(x)\right\} \cdot \prod_{j \in I_{c d}} P\left\{V_{n j} \leqq w_{n}(y)\right\} .
$$

Of course, $\log p=\log [1-(1-p)]<-(1-p)$ for $0<p<1$, so the logarithm of the displayed product is at most

$$
-\sum_{j \in I_{a b}} P\left\{V_{n j}>w_{n}(x)\right\}-\sum_{j \in I_{c d}} P\left\{V_{n j}>w_{n}(y)\right\} .
$$

Due to the minus-sign, $P\left\{V_{n j}>w_{n}(x)\right\}$ must be estimated from below. Recall $\lambda_{n j}(x)$ and $\psi_{n j}(x)$ from (2.8) and (2.23). Then

$$
\begin{aligned}
P\left\{V_{n j}>w_{n}(x)\right\} & =P\left\{\left\{N_{j}>k p_{j}+\sqrt{k p_{j}} \sqrt{2 \log \frac{1}{\varepsilon}} \lambda_{n j}(x)\right\}\right. \\
& =[1+o(1)] \psi_{n j}(x),
\end{aligned}
$$

the " $o(1)$ " being uniform over $j$ with $\left|t_{n j}-t_{n}\right|<\delta$, provided $\delta$ is small. The argument for (3.6) is omitted, being very similar to one below. The lim sup of the probability in (3.4) is then at most $\exp \{Q(x, y)\}$, by (2.27). Further details on the lim sup are omitted.

For the lim inf,

Under the conditions of (3.4), the probability in (3.4) is bounded below by $\gamma+o(1)$, where $\log \gamma$ is in turn bounded below by

$$
-[1+o(1)]\left[\sum_{j \in I_{a b}} \psi_{n j}(x)+\sum_{j \in I_{c d}} \psi_{n j}(y)\right]+o(1) .
$$

Granting (3.7), an appeal to (2.27) shows that the lim inf of the probability in $(3.4)$ is at least $\exp \{Q(x, y)\}$. This completes the proof of (3.4), and hence of the main theorem (1.24).

Thus, (1.24) is reduced to (3.7). Now begins a series of calculations designed to prove (3.7). Eventually, lemma (3.2) of [1] will be used. Let

$$
\begin{aligned}
A_{j} & =\left\{V_{n j} \leqq w_{n}(x)\right\} \quad \text { for } \quad j \in I_{a b} \\
& =\left\{V_{n j} \leqq w_{n}(y)\right\} \text { for } j \in I_{c d}
\end{aligned}
$$

$K_{j}$ be the set of $i$ with $t_{n}-\delta \leqq t_{n i} \leqq t_{n j}$

$$
\begin{gathered}
g_{j}=\sum_{i \in K_{j}} p_{i} \\
R=2\left(k \log \frac{1}{\varepsilon}\right)^{1 / 2} \\
G_{j}=\left\{\sum_{i \in K_{j}} N_{i}>k g_{j}-R\right\}
\end{gathered}
$$


In (3.9), note that $t_{n i} \leqq t_{n j}$ iff $i \leqq j . \quad$ In (3.10), assumption (1.16) implies

$$
\lim \sup _{n} \max _{j}\left\{g_{j}: t_{n j} \in I\right\}<1
$$

LeMma 3.14. $\sum_{j}\left\{1-P\left(G_{j}\right): t_{n j} \in I\right\} \rightarrow 0$ as $n \rightarrow \infty$.

Proof. Clearly, $\sum_{i \in K_{j}} N_{i}$ is binomial, with number of trials $k$ and success probability $g_{j}<1$. So

$$
\begin{aligned}
1-P\left(G_{j}\right) & <\exp \left\{-\frac{1}{2} R^{2} / k g_{j}\right\} \\
& <\exp \left\{-\frac{1}{2} R^{2} / k\right\} \\
& =\exp \left\{-2 \log \frac{1}{\varepsilon}\right\} \text { by } \\
& =\varepsilon^{2}
\end{aligned}
$$

But there are only $o(1 / \varepsilon)$ indices $j$ with $t_{n j} \in I$, completing the proof. The version of Bernstein's inequality used above appears as theorem (4) in [5].

Lemma 3.15. Let $N_{j}^{\prime}$ be binomial, with success probability $p_{j}^{\prime}=$ $p_{j} /\left(1-g_{j-1}\right)$ and number of trials $k_{j}^{\prime}$, the integer part of $k\left(1-g_{j-1}\right)+$ R. Let

$$
\begin{aligned}
V_{j}^{\prime} & =V_{n j}^{\prime}=\alpha_{n j}\left(k p_{j}\right)^{-1 / 2}\left(N_{j}^{\prime}-k p_{j}\right)+\beta_{n j}\left(2 \log \frac{1}{\varepsilon}\right)^{1 / 2} \pi_{j}(x) \\
& =\pi_{n j}(x)=P\left\{V_{j}^{\prime n}>w_{n}(x)\right\} .
\end{aligned}
$$

If $\delta$ is small and $n$ is large, then

$$
P\left\{M_{a b} \leqq w_{n}(x) \text { and } \quad M_{c d} \leqq w_{n}(y)\right\}
$$

is bounded below by

$$
\left\{\prod_{j \in I_{a b}}\left[1-\pi_{j}(x)\right]\right\} \cdot\left\{\prod_{j \in I_{c d}}\left[1-\pi_{j}(y)\right]\right\}-\sum_{j \in I_{-\infty} \infty}\left[1-P\left(G_{j}\right)\right] .
$$

By definition (2.6), $I_{-\infty \infty}$ is the set of $j$ 's with $\left|t_{n j}-t_{n}\right|<\delta$.

Proof. This follows from (3.2) of [1]. Indeed, let $\mathscr{F}_{j}$ be the $\sigma$-field spanned by $N_{i}$ for $i \in K_{j}$. Given $\mathscr{F}_{j-1}$, the variable $N_{j}$ is conditionally binomial, with success probability $p_{j}^{\prime}$; the number of trials $T_{j}$ is an $\mathscr{F}_{j-1}$-measurable random variable:

$$
T_{j}=k-\sum_{i \in K_{j-1}} N_{i}
$$


On $G_{j-1}$, however, $T_{j} \leqq k_{j}^{\prime}$. So

$$
\begin{aligned}
P\left\{V_{j}\right. & \left.\leqq w_{n}(x) \mid \mathscr{F}_{j-1}\right\} \\
& =P\left\{N_{j} \leqq k p_{j}+\sqrt{k p_{j}} \sqrt{2 \log \frac{1}{\varepsilon}} \lambda_{n j}(x) \mid \mathscr{F}_{j-1}\right\} \\
& \geqq P\left\{N_{j}^{\prime} \leqq k p_{j}+\sqrt{k p_{j}} \sqrt{2 \log \frac{1}{\varepsilon}} \lambda_{n j}(x)\right\} \\
& =P\left\{V_{j}^{\prime} \leqq w_{n}(x)\right\} \\
& =1-\pi_{j}(x) .
\end{aligned}
$$

The heuristic: the more often you toss the coin, the more heads come up.

In this argument, it is tacitly supposed that $\alpha_{n j}>0$, which will be the case for all $j$ with $\left|t_{n j}-t_{n}\right|<\delta$, if $\delta$ is small and $n$ is large.

This proves the first part of (3.7): the product of the $(1-\pi)$ 's in (3.15) serves for $\gamma$, and the sum of the $\left[1-P\left(G_{j}\right)\right]$ 's is $o(1)$ by (3.14). For the second part of (3.7), estimate

$$
\log \gamma \doteq-\sum_{j \in I_{a b}} \pi_{j}(x)-\sum_{j \in I_{c d}} \pi_{j}(y) .
$$

This will now be made rigorous.

Lemma 3.16. If $\delta$ is small, then

$$
\pi_{j}(x)=[1+o(1)] \psi_{n j}(x)
$$

uniformly over $j$ with $\left|t_{n j}-t_{n}\right|<\delta$, as $n \rightarrow \infty$.

Proof. To begin with, for some $\zeta$ with $0 \leqq \zeta<1$,

$$
k_{j}^{\prime}=k\left(1-g_{j-1}\right)+R-\zeta,
$$

so

$$
k_{j}^{\prime} p_{j}^{\prime}=k p_{j}+\frac{(R-\zeta) p_{j}}{1-g_{j-1}}
$$

and

$$
k_{j}^{\prime} p_{j}^{\prime}=k p_{j}+0\left(R p_{j}\right)
$$

by (3.13). Consequently,

$$
\begin{aligned}
\left(k_{j}^{\prime} p_{j}^{\prime}\right) /\left(k p_{j}\right) & =1+O\left[\left(\log \frac{1}{\varepsilon}\right)^{1 / 2} / k^{1 / 2}\right] \text { by } \\
& =1+o\left(1 / \log \frac{1}{\varepsilon}\right) \text { by }(1.20) .
\end{aligned}
$$


Of course, $\pi_{j}(x)$ is the chance that $N_{j}^{\prime}$ exceeds

$$
k p_{j}+\sqrt{k p_{j}} \sqrt{2 \log \frac{1}{\varepsilon}} \lambda_{n j}(x) .
$$

Now, the expression (3.19) can be rewritten as

$$
k_{j}^{\prime} p_{j}^{\prime}+\sqrt{k_{j}^{\prime} p_{j}^{\prime}} \sqrt{2 \log \frac{1}{\varepsilon}}\left\{\lambda_{n j}(x)+o\left(1 / \log \frac{1}{\varepsilon}\right)\right\} .
$$

Granting this transformation, proposition (3.17) of [1] can be applied and completes the proof. The conditions of that lemma are satisfied by assumptions (1.2) and (1.17-18), and the properties of $k_{j}^{\prime}$ and $p_{j}^{\prime}$ developed obove. Note that $\lambda_{n j}(x)$ is nearly 1 , by (2.16a). Also, (3.4) of [1] can be used to simplify the expression in (3.17) of [1].

To get from (3.19) to (3.20), note that

$$
\begin{aligned}
\frac{k p_{j}-k_{j}^{\prime} p_{j}^{\prime}}{\sqrt{k_{j}^{\prime} p_{j}^{\prime}} \sqrt{2 \log \frac{1}{\varepsilon}}} & \approx \frac{k p_{j}-k_{j}^{\prime} p_{j}^{\prime}}{\sqrt{k p_{j}} \sqrt{2 \log \frac{1}{\varepsilon}}} & & \text { by }(3.18) \\
& =O\left(\sqrt{p_{j}}\right) & & \text { by }(3.11) \text { and }(3.17) \\
& =o\left(1 / \log \frac{1}{\varepsilon}\right) & & \text { by }(1.17)
\end{aligned}
$$

So, it is harmless to replace the first term $k p_{j}$ in (3.19) by $k_{j}^{\prime} p_{j}^{\prime}$.

Now replace the factor $\sqrt{k p_{j}}$ in the second term of (3.19) by $\sqrt{k_{j}^{\prime} p_{j}^{\prime}}$. The error is $\gamma_{1} \gamma_{2} \gamma_{3}$, where

$$
\begin{aligned}
& \gamma_{1}=\sqrt{k_{j}^{\prime} p_{j}^{\prime}} \sqrt{2 \log \frac{1}{\varepsilon}} \\
& \gamma_{2}=\left[\left(k p_{j}\right) /\left(k_{j}^{\prime} p_{j}^{\prime}\right)\right]^{1 / 2}-1 \\
& \gamma_{3}=\lambda_{n j}(x)
\end{aligned}
$$

But $\gamma_{2}=o(1 / \log 1 / \varepsilon)$ by (3.18), and $\gamma_{3}=O(1)$ by (2.16a). This completes the move from (3.19) to (3.20), and hence the proof.

Proof of 3.7. The probability in (3.14) is bounded below by $\gamma-\gamma^{\prime}$, where

$$
\begin{aligned}
& \gamma=\prod_{j \in I_{a b}}\left[1-\pi_{j}(x)\right] \cdot \prod_{j \in I_{c d}}\left[1-\pi_{j}(y)\right] \\
& \gamma^{\prime}=\sum_{j \in I_{-\infty}}\left[1-P\left(G_{j}\right)\right]
\end{aligned}
$$

in view of (3.15). And $\gamma^{\prime} \rightarrow 0$ by (3.14). Next,

$$
\pi_{j}(x)=[1+o(1)] \psi_{n j}(x)=o(1)
$$


uniformly over $j$ with $\left|t_{n j}-t_{n}\right|<\delta$, by (3.16) and (2.24). Of course, $\log (1-u)=-[1+o(1)] u$ as $u \rightarrow 0$, and this completes the proof.

This completes the proof of the distributional assertions in (1.24), and it remains only to prove that the maximum occurs at a unique index. First, some heuristics. Inspection of (1.24) suggests that the maximum is likely to occur only for $O((1 / \varepsilon) / \sqrt{\log 1 / \varepsilon})$ indices $j$ near $c_{n}+\varepsilon_{n}^{-1} t_{n}$. Call these the critical $j$ 's. If $N_{j}$ is maximal, it must take a critical value $i$, of order

$$
k p_{j}+\sqrt{k p_{j}} \sqrt{2 \log \frac{1}{\varepsilon}} \lambda_{n j}(x),
$$

where

$$
\lambda_{n j}(x) \doteq 1+\frac{1}{2} \rho^{2}\left(t_{n j}-t_{n}\right)^{2}-\frac{1}{2} \frac{\log \log 1 / \varepsilon}{\log 1 / \varepsilon}+\frac{1}{4} \frac{x}{\log 1 / \varepsilon} .
$$

In particular, there are $O\left[\sqrt{k p_{j}} / \sqrt{\log 1 / \varepsilon}\right]$ values $i$ which are critical for the index $j$.

Suppose the maximum occurs at indices $j$ and $j^{\prime}$. Then

$$
N_{j}=i \text { and } N_{j^{\prime}}=i^{\prime}
$$

with

$$
\begin{aligned}
& \alpha_{n j} \frac{i-k p_{j}}{\sqrt{k p_{j}}}+\beta_{n j} \sqrt{2 \log \frac{1}{\varepsilon}} \\
& =\alpha_{n j^{\prime}} \frac{i^{\prime}-k p_{j^{\prime}}}{\sqrt{k \rho_{j^{\prime}}}}+\beta_{n j^{\prime}} \sqrt{2 \log \frac{1}{\varepsilon}} .
\end{aligned}
$$

Thus, $i$ and $i^{\prime}$ are rigidly linked. The chance that the maximum occurs at two distinct indices $j$ and $j^{\prime}$ is then

$$
\sum_{j, j^{\prime}} \sum_{i, i^{\prime}} P\left\{N_{j}=i \text { and } N_{j^{\prime}}=i^{\prime}\right\}
$$

This sum extends only over critical indices $j$ and $j^{\prime}$; for each $j$, the inner sum is over $i$ 's critical for $j$. Now $N_{j}$ and $N_{j^{\prime}}$ are nearly independent, so

$$
P\left\{N_{j}=i \quad \text { and } \quad N_{j^{\prime}}=i^{\prime}\right\} \doteq P\left\{N_{j}=i\right\} \cdot P\left\{N_{j}^{\prime}=i^{\prime}\right\} .
$$

The probabilities on the right can be estimated by (3.10) of [1]:

$$
\begin{aligned}
P\left\{N_{j}=i\right\} & \doteq \frac{1}{\sqrt{2 \pi k p_{j}}} \exp \left\{-\frac{1}{2} \frac{\left(i-k p_{j}\right)^{2}}{k p_{j}}\right\} \\
& \sim \frac{1}{\sqrt{k p_{j}}} \varepsilon\left(\log \frac{1}{\varepsilon}\right) .
\end{aligned}
$$

See (2.11). Likewise, 


$$
P\left\{N_{j^{\prime}}=i^{\prime}\right\} \sim \frac{1}{\sqrt{k p_{j^{\prime}}}} \varepsilon\left(\log \frac{1}{\varepsilon}\right) .
$$

In the inner sum of (3.27), the index $i$ determines $i^{\prime}$ by (3.26); so the inner sum is

$$
O\left[\frac{\sqrt{k p_{j}}}{\sqrt{\log 1 / \varepsilon}} \cdot \frac{1}{\sqrt{k p_{j}}} \cdot \frac{1}{\sqrt{k p_{j^{\prime}}}} \varepsilon^{2}\left(\log \frac{1}{\varepsilon}\right)^{2}\right] .
$$

But $k p_{j^{\prime}} \gg(\log 1 / \varepsilon)^{3}$ by $(1.19)$, so the inner sum is $o\left(\varepsilon^{2}\right)$. Coming to the outer sum, the number of terms is $O\left(\left(1 / \varepsilon^{2}\right) / \log 1 / \varepsilon\right)$.

Returning to rigorous argument, the main estimate is the following.

Lemma 3.28. Assume (1.1)-(1.23). Fix positive, finite numbers $a$ and $b$. Then, uniformly over pairs of indices $j \neq j^{\prime}$ in $I_{-a a}$, and values $i, i^{\prime}$ satisfying

$$
\begin{aligned}
k p_{j}+ & \sqrt{k p_{j}} \sqrt{2 \log \frac{1}{\varepsilon}} \lambda_{n j}(-b) \\
& \leqq i \leqq k p_{j}+\sqrt{k p_{j}} \sqrt{2 \log \frac{1}{\varepsilon}} \lambda_{n j}(b) \\
k p_{j^{\prime}} & +\sqrt{k p_{j^{\prime}}} \sqrt{2 \log \frac{1}{\varepsilon}} \lambda_{n j^{\prime}}(-b) \\
& \leqq i^{\prime} \leqq k p_{j^{\prime}}+\sqrt{k p_{j^{\prime}}} \sqrt{2 \log \frac{1}{\varepsilon}} \lambda_{n j^{\prime}}(b)
\end{aligned}
$$

we have

$$
\begin{gathered}
P\left\{N_{j}=i\right\}=O\left[\left(k p_{j}\right)^{-1 / 2} \in \log \frac{1}{\varepsilon}\right] \\
P\left\{N_{j^{\prime}}=i^{\prime} \mid N_{j}=i\right\}=O\left[\left(k p_{j^{\prime}}\right)^{-1 / 2} \varepsilon \log \frac{1}{\varepsilon}\right] .
\end{gathered}
$$

Proof. The first assertion (3.31) is more or less immediate from (3.10) of [1], and the present (2.16). Details are omitted; also see (3.4) of [1]. For the second assertion (3.32), given $N_{j}=i$, the conditional distribution of $N_{j^{\prime}}$ is binomial with success probability $\hat{p}=$ $p_{j^{\prime}} /\left(1-p_{j}\right)$ and number of trials $\hat{k}=k-i$. Some preliminary estimates are needed before appealing to (3.10) of [1]. All " $O$ " and " $O$ " estimates are uniform over $j, j^{\prime}, i$ and $i^{\prime}$ satisfying the conditions of the lemma.

It will be shown that 


$$
\begin{aligned}
\hat{k} \hat{p} & =k p_{j^{\prime}}\left[1+O\left(k^{-1 / 2}\left(\log \frac{1}{\varepsilon}\right)^{-3 / 2}\right)\right] \\
& =k p_{j^{\prime}}\left[1+o\left(1 / \log \frac{1}{\varepsilon}\right)\right]
\end{aligned}
$$

$$
\left(i^{\prime}-\hat{k} \hat{p}\right)^{2} / \hat{p} \hat{k}=2 \log \frac{1}{\varepsilon}-2 \log \log \frac{1}{\varepsilon}+O(1) .
$$

Granting (3.33)-(3.34), the second assertion too follows from (3.10) of [1].

Turn now to proving (3.33)-(3.34). First,

$$
\hat{k} \hat{p}-k p_{j^{\prime}}=\left(k p_{j}-i\right) p_{j^{\prime}} /\left(1-p_{j}\right) \text {. }
$$

Now $p_{j} \rightarrow 0$ by $(1.17)$, so $1 /\left(1-p_{j}\right)=O(1)$. Next, $\lambda_{n j}(x)=O(1)$ uniformly over $j$ with $\left|t_{n j}-t_{n}\right|<\delta$ and $x$ with $|x| \leqq b$, by (2.16a). So

$$
i-k p_{j}=O\left[\sqrt{k p_{j}} \sqrt{2 \log \frac{1}{\varepsilon}}\right]
$$

and

$$
\left(\hat{k} \hat{p}-k p_{j^{\prime}}\right) / k p_{j^{\prime}}=O\left[\sqrt{p_{j^{\prime}}} \sqrt{\log \frac{1}{\varepsilon}} / \sqrt{k}\right] .
$$

Now (1.20) proves (3.33).

For (3.34),

$$
i^{\prime}-\hat{k} \hat{p}=\left(i^{\prime}-k p_{j^{\prime}}\right)+\left(k p_{j^{\prime}}-\hat{k} \hat{p}\right)
$$

so

$$
\left(i^{\prime}-\hat{k} \hat{p}\right)^{2}=\left(i^{\prime}-k p_{j^{\prime}}\right)^{2}+2\left(i^{\prime}-k p_{j^{\prime}}\right)\left(k p_{j^{\prime}}-\hat{k} \hat{p}\right)+\left(k p_{j^{\prime}}-\hat{k} \hat{p}\right)^{2} .
$$

Now

$$
\begin{aligned}
\left(i^{\prime}-k p_{j^{\prime}}\right)^{2} / \hat{k} \hat{p} & =\left[\left(i^{\prime}-k p_{j^{\prime}}\right)^{2} / k p_{j^{\prime}}\right]\left[1+o\left(1 / \log \frac{1}{\varepsilon}\right)\right] \\
& =\left[2 \log \frac{1}{\varepsilon}-2 \log \log \frac{1}{\varepsilon}+O(1)\right]\left[1+o\left(1 / \log \frac{1}{\varepsilon}\right)\right] \\
& =2 \log \frac{1}{\varepsilon}-2 \log \log \frac{1}{\varepsilon}+O(1)
\end{aligned}
$$

where (3.33) was used in the first line, condition (3.30) and estimate $(2.16 \mathrm{~b}-\mathrm{c})$ of $\lambda_{n j}$ in the second. Likewise,

$$
\begin{aligned}
\left(i^{\prime}-k p_{j^{\prime}}\right)\left(k p_{j^{\prime}}-\hat{k} \hat{p}\right) / \hat{k} \hat{p} & \sim\left(i^{\prime}-k p_{j^{\prime}}\right)\left(k p_{j^{\prime}}-\hat{k} \hat{p}\right) / k p_{j^{\prime}} \text { by }(3.33) \\
& \sim \sqrt{p_{j^{\prime}} p_{j^{\prime}}} \cdot \log \frac{1}{\varepsilon} \text { by }(3.35-6) \\
& \rightarrow 0 \text { by }(1.17)
\end{aligned}
$$


Finally,

$$
\begin{aligned}
\left(k p_{j^{\prime}}-\hat{k} \hat{p}\right)^{2} / \hat{k} \hat{p} & \sim\left(k p_{j^{\prime}}-\hat{k} \hat{p}\right)^{2} / k p_{j^{\prime}} \quad \text { by }(3.33) \\
& \sim p_{j^{\prime}} p_{j} \log \frac{1}{\varepsilon} \text { by }(3.36) \\
& \sim 0 \text { by }(1.17)
\end{aligned}
$$

4. A generalization. Some cases of interest do not quite fit into the framework of conditions (1.4-15). Assume (1.1-3), for $j$ 's such that $t_{n j}$ falls in the compact interval $I$. Let $t_{n}$ and $t_{\infty}$ be interior points of $I$, with $t_{n} \rightarrow t_{\infty}$. Let $\alpha_{n}, \alpha_{n}^{\prime}$ and $\beta_{n}$, $\beta_{n}^{\prime}$ be numbers, for $1 \leqq n<\infty$; let $\alpha_{\infty}^{\prime \prime}$ and $\beta_{\beta}^{\prime \prime}$ be numbers. For any positive $\eta_{1}$ and $\eta_{2}$, suppose there exist positive, finite numbers $\delta_{1}=\delta_{1}\left(\eta_{1}, \eta_{2}\right)$ and $n^{*}=n^{*}\left(\eta_{1}, \eta_{2}\right)$ such that $\left|t_{n j}-t_{n}\right|<\delta_{1}$ and $n>n^{*}$ entail

$$
\begin{aligned}
\mid \alpha_{n j} & -\alpha_{n}-\alpha_{n}^{\prime} \cdot\left(t_{n j}-t_{n}\right)-\frac{1}{2} \alpha_{\infty}^{\prime \prime} \cdot\left(t_{n j}-t_{n}\right)^{2} \mid \\
& <\eta_{1} \cdot\left(t_{n j}-t_{n}\right)^{2}+\eta_{2} / \log \frac{1}{\varepsilon_{n}} \\
\mid \beta_{n j} & -\beta_{n}-\beta_{n}^{\prime} \cdot\left(t_{n j}-t_{n}\right)-\frac{1}{2} \beta_{\infty}^{\prime \prime}\left(t_{n j}-t_{n}\right)^{2} \mid \\
& <\eta_{1} \cdot\left(t_{n j}-t_{n}\right)^{2}+\eta_{2} / \log \frac{1}{\varepsilon_{n}}
\end{aligned}
$$

Suppose furthermore

$$
\begin{gathered}
\alpha_{n} \rightarrow \alpha_{\infty} \text { positive, and } \alpha_{n}^{\prime} \rightarrow \alpha_{\infty}^{\prime} \text {, both finite } \\
\alpha_{n}^{\prime}+\beta_{n}^{\prime}=0 \\
\alpha_{\infty}^{\prime \prime}+\beta_{\beta}^{\prime \prime}<0 .
\end{gathered}
$$

Let

$$
\rho^{2}=-\left(\alpha_{\infty}^{\prime \prime}+\beta_{\infty}^{\prime \prime}\right) / \alpha_{\infty}>0 \text {. }
$$

THEOREM 4.7. Suppose (1.1-3) and (1.16-23), but (4.1-5) in place of (1.4-15). Let $n \rightarrow \infty$. There is some small positive $\delta$ such that, with probability approaching one, $M_{n}=\max _{j}\left[V_{n j}:\left|t_{n j}-t_{n}\right|<\delta\right]$ is assumed at a unique index $L_{n}$. Furthermore, the chance that

$$
\rho \sqrt{2 \log \frac{1}{\varepsilon_{n}}}\left[\varepsilon_{n}\left(L_{n}-c_{n}\right)-t_{n}\right]<y
$$

and

$$
M_{n}<\alpha_{n} w_{n}(x)+\beta_{n} \sqrt{2 \log \frac{1}{\varepsilon_{n}}}
$$


converges to

$$
\Phi(y) \cdot \exp \left[-\frac{1}{2 \rho} e^{-(1 / 2) x}\right]
$$

The prrof is omitted, being identical to that for (1.24). Note, however, that (4.7) gives no control over $j$ 's with $t_{n j}$ remote from $t_{\infty}$. If, for example, $\alpha_{n j}=\alpha\left(t_{n j}\right)$ and $\beta_{n j}=\beta\left(t_{n j}\right)$ where $\alpha$ and $\beta$ are smooth functions, then (4.7) can be used separately in the vicinity of each local maximum of $\alpha+\beta$.

\section{REFERENCES}

1. P. Diaconis and D. Freedman, On the maximum difference between the empirical and expected histograms for sums, Pacific J. Math., 100 (1982a), 287-327.

2. - On the mode of an empirical histogram for sums., Pacific J. Math., 100 (1982d), 373-385.

3. - On the maximum difference between a histogram for data and the underlying density, Z. Wahrscheinlichkeitstheorie, 58 (1981), 139-169.

4. - On the maximum difference between the empirical and expected histograms for sums, Part II, Pacific J. Math., 100 (1982c), 359-371.

5. D. Freedman, Another note on the Borel-Cantelli lemma and the strong law, with the Poisson approximation as a by-product, Ann. Prob., 1, No. 6, (1973), 920-925.

6. C. Mallows, An inequality involving multinomial probabilities, Biometrika, 55 (1968), 422-424.

Received January 7, 1981. Research partially supported by National Science Foundation Grant MCS-77-01665.

UNIVERSity of CALIFornia

Berkeley, CA 94720 


\section{PACIFIC JOURNAL OF MATHEMATICS}

\section{EDITORS}

DONALD BABBITT (Managing Editor)

University of California

Los Angeles, CA 90024

Hugo RossI

University of Utah

Salt Lake City, UT 84112

C. C. Moore and Arthur Agus

University of California

Berkeley, CA 94720
J. DugundJI

Department of Mathematics

University of Southern California

Los Angeles, CA 90007

R. FinN and J. MiLgRAM

Stanford University

Stanford, CA 94305

\section{ASSOCIATE EDITORS}
R. ARENS
E. F. BECKENBACH
B. H. NeumanN
F. WOLF
K. YoSHIDA

\section{SUPPORTING INSTITUTIONS}

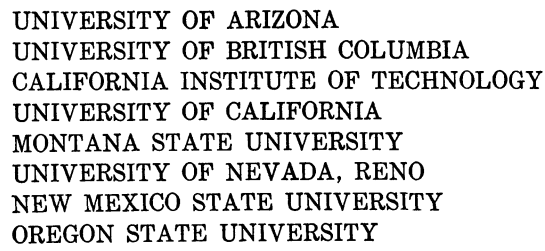

UNIVERSITY OF ARIZONA

UNIVERSITY OF BRITISH COLUMBIA CALIFORNIA INSTITUTE OF TECHNOLOGY UNIVERSITY OF CALIFORNIA MONTANA STATE UNIVERSITY

UNIVERSITY OF NEVADA, RENO NEW MEXICO STATE UNIVERSITY OREGON STATE UNIVERSITY

\author{
UNIVERSITY OF OREGON \\ UNIVERSITY OF SOUTHERN CALIFORNIA \\ STANFORD UNIVERSITY \\ UNIVERSITY OF AAWAII \\ UNIVERSITY OF TOKYO \\ UNIVERSITY OF UTAH \\ WASHINGTON STATE UNIVERSITY \\ UNIVERSITY OF WASHINGTON
}

The Supporting Institutions listed above contribute to the cost of publication of this Journal, but they are not owners or publishers and have no responsibility for its content or policies,

Mathematical parers intended for publication in the Pacific Journal of Mathematics should be in typed form or offset-reproduced, (not dittoed), double spaced with large margins. Please do not use built up fractions in the text of the manuscript. However, you may use them in the displayed equations. Underline Greek letters in red, German in green, and script in blue. The first paragraph or two must be capable of being used separately as a synopsis of the entire paper. Please propose a heading for the odd unmbered pages of less than 35 characters. Manuscripts, in triplicate, may be sent to any one of the editors. Please classify according to the scheme of Math. Reviews, Index to Vol. 39. Supply name and address of author to whom proofs should be sent. All other communications should be addressed to the managing editor, or Elaine Barth, University of California, Los Angeles, California, 90024 .

50 reprints to each author are provided free for each article, only if page charges have been substantially paid. Additional copies may be obtained at cost in multiples of 50 .

The Pacific Journal of Mathematics is issued monthly as of January 1966, Regular subscription rate: $\$ 114.00$ a year (6 Vol., 12 issues). Special rate: $\$ 57.00$ a year to individual members of supporting institution.

Subscriptions, orders for numbers issued in the last three calendar years, and changes of address shoud be sent to Pacific Journal of Mathematics, P.O. Box 969, Carmel Valley, CA 93924, U.S.A. Old back numbers obtainable from Kraus Periodicals Co., Route 100, Millwood, NY 10546.

\section{PUBLISHED BY PACIFIC JOURNAL OF MATHEMATICS, A NON-PROFIT CORPORATION}

Printed at Kokusai Bunken Insatsusha (International Academic Printing Co., Ltd.). 8-8, 3-chome, Takadanobaba, Shinjuku-ku, Tokyo 160, Japan.

Copyright (C) 1982 by Pacific Journal of Mathematics Manufactured and first issued in Japan 


\section{Pacific Journal of Mathematics}

Vol. 100, No. $2 \quad$ October, 1982

Kenneth F. Andersen, On the transformation of Fourier coefficients of

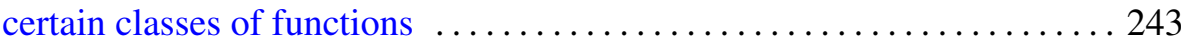

Steven Albert Bleiler, Realizing concordant polynomials with prime knots

Reinhard Bürger, Functions of translation type and solid Banach spaces of functions

Ulrich Daepp, The saturation of $k$-analytic rings and topological equivalence of associated analytic set germs .................. 271

Persi W. Diaconis and David Amiel Freedman, On the maximum difference between the empirical and expected histograms for sums . . . 287

David Amiel Freedman, On the maximum of scaled multinomial

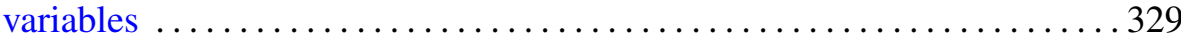

Persi W. Diaconis and David Amiel Freedman, On the difference between the empirical histogram and the normal curve, for sums. II ......... 359

Persi W. Diaconis and David Amiel Freedman, On the mode of an

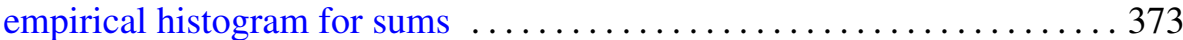

Jutta Hausen, Supplemented modules over Dedekind domains 387

Elyahu Katz, A moduli representation for the classification of twisted tensor

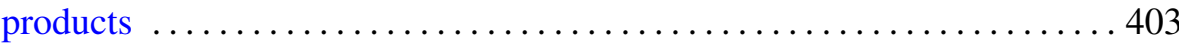

H. C. Madhekar and N. K. Thakare, Biorthogonal polynomials suggested by the Jacobi polynomials

Ted R. Pettis, Collections of covers of metric spaces 425

Ryōtarō Satō, Maximal functions for a semiflow in an infinite measure space

Michael Jay Stob, Invariance of properties under automorphisms of the lattice of recursively enumerable sets 